\title{
LE DOUBLE DIVIDENDE LES APPROCHES THÉORIQUES
}

\author{
Mireille CHIROLEU-ASSOULINE
}

Université de Paris 1, EUREQua et ERASME

\author{
EUREQua \\ Université de Paris I Panthéon-Sorbonne \\ Maison des Sciences Économiques \\ 106-112 bd de l'Hôpital \\ 75647 PARIS Cedex 13. \\ Tél : 0144078224 \\ Fax : 01440782 \\ ERASME \\ École Centrale Paris \\ Grande Voie des Vignes \\ 92295 CHATENAY-MALABRY Cedex \\ Tél : 0141131179 \\ Fax : 0141131667 \\ e-mail : assoulin@univ-paris1.fr
}




\section{RÉSUMÉ : Le double dividende - Les approches théoriques}

La notion de double dividende se réfère au cas où l'instauration d'une taxe environnementale, à recettes budgétaires inchangées pour l'État, fait apparaitre à la fois un bénéfice environnemental et un bénéfice de nature économique. Les premières études théoriques, en équilibre général, réfutaient cette hypothèse de double dividende et montraient qu'au contraire, les taxes environnementales exacerbent, plutôt qu'elles n'allègent, les distorsions fiscales préexistantes. Cependant, le rôle essentiel joué dans ces résultats par l'élasticité non compensée de l'offre de travail au pouvoir d'achat du salaire met l'accent sur les hypothèses fondamentales de ces études, à savoir la concurrence pure et parfaite et l'équilibre sur le marché du travail. Les imperfections de concurrence ainsi que l'existence de chômage involontaire rendent plus probable l'occurrence d'un double dividende au sens fort, de même que la prise en compte du rôle de la qualité de l'environnement dans les comportements des ménages ou dans la structure productive.

\section{ABSTRACT : The double dividend - Theoretical approaches}

One speaks about double dividend when the revenue neutral substitution of environmental taxes for other taxes leads to both an environmental benefit and an economic one. The first theoretical studies of this question, in a general equilibrium framework, assess that there is no secondary benefit in the sense of a reduction in tax distorsions. However, there are cases in which positive welfare gains obtain: when the environmental tax reform involves a tax shifting from labor to other factors or when there are feedbacks effects from environmental quality to the economic system. When studying this issue under the assumptions of imperfect competition and non clearing labour market, the occurrence of a double dividend becomes more likely. 


\section{LE DOUBLE DIVIDENDE}

\section{LES APPROCHES THÉORIQUES}

\section{Introduction : La problématique de l'antagonisme entre qualité de l'environnement et économie (croissance, emploi...) et les premières évaluations empiriques}

Le débat sur le double dividende est né de la polémique autour de l'ampleur de l'effet de serre additionnel, de l'analyse de ses causes et de l'évaluation de ses conséquences. En effet, la décision de mettre en œuvre une politique environnementale de réduction des émissions polluantes (dioxyde de carbone, etc...) correspond au souci d'internaliser des externalités provoquant des dommages environnementaux dont la mesure, dans le cas de l'effet de serre, est ainsi affectée d'une forte incertitude. Cette incertitude remet en cause la possibilité d'une internalisation totale et conduit même certains à mettre en doute l'opportunité même des politiques environnementales. Il a donc semblé utile d'évaluer les coûts économiques bruts de ces politiques, et plus particulièrement des taxes environnementales (écotaxes, taxes sur le carbone ou sur l'énergie), c'est-à-dire les coûts économiques à l'exclusion des bénéfices économiques, potentiels mais contestés, associés aux dommages environnementaux évités. Ces coûts constituent ainsi une borne inférieure à ce que devraient être les bénéfices environnementaux pour justifier, du point de vue de l'efficacité, la mise en œuvre de ces politiques.

Par rapport à d'autres instruments économiques permettant d'internaliser les externalités, l'un des avantages de la taxe environnementale, par rapport aux subventions, quotas ou marchés de droits à polluer, est de procurer un revenu à l'Etat, revenu qui peut ensuite être réinjecté dans l'économie. Terkla [1984] fut l'un des premiers à avancer que ce recyclage du revenu pourrait réduire, voire annuler le coût brut de mise en œuvre des taxes environnementales (argument repris par Lee et Misiolek [1986], Parry [1995] ou Poterba [1993]). En effet, les premières évaluations des politiques environnementales avaient été réalisées sans se préoccuper du recyclage éventuel du produit de la taxe, ce qui aboutissait à des estimations particulièrement élevées des coûts de ces politiques en 
termes d'activité et d'emploi. Dans un deuxième temps, les analyses de l'influence des taxes environnementales reposèrent sur l'hypothèse implicite d'une redistribution forfaitaire de leur rendement, afin de ne pas provoquer de distorsions supplémentaires. Les coûts bruts des taxes environnementales se révélèrent dans cette hypothèse naturellement plus faibles qu'en l'absence de redistribution. Enfin Terkla [1984], Baumol et Oates [1988], Pearce [1991], Oates [1991] ou Poterba [1993] suggérèrent qu'il pouvait être plus efficace, au sens de Pareto, de substituer des taxes sur la pollution à d'autres taxes socialement plus coûteuses. Pearce [1991] affirme ainsi que la substitution de taxes environnementales à des taxes distordantes peut produire un double dividende en (1) décourageant les activités qui dégradent l'environnement et en (2) réduisant le coût de distorsion du système fiscal.

Les premières estimations dans ce sens estimèrent significatifs les potentiels de gains en efficacité (Terkla [1984]). Un certain nombre d'études appuyées sur des simulations de modèles macroéconométriques, de structure néo-keynésienne, firent apparaître la possibilité de coûts bruts négatifs de l'écotaxe et le terme de double dividende fut alors utilisé pour désigner un bénéfice autre qu'environnemental, sous la forme d'une stimulation de la croissance et de l'emploi (EC[1992] et [1994], Beaumais et Godard [1994], Lemiale et Zagamé [1998]). Cette possibilité d'apparition d'un double dividende fonde l'élaboration de stratégies sans regret (no regret strategy) correspondant à “ des mesures qui, même si le risque global se révèle finalement infondé, ont un intérêt propre et un coût économique nul, voire négatif" (Beaumais, Schubert et Zagamé [1998]). Les modalités d'obtention de ce double dividende apparaissaient différentes selon les pays, en fonction des systèmes fiscaux en vigueur : ainsi il pourrait être obtenu aux États-Unis grâce au recyclage du rendement d'une taxe sur l'énergie par une diminution des taxes sur le capital (GIEC [1997]) tandis qu'en Europe et en particulier en France il se produirait grâce à un recyclage par une réduction des charges sociales sur le travail (CGP [1993]).

En suivant Goulder [1994], on peut noter que le débat autour de l'éventualité d'un second dividende reflète essentiellement les incertitudes planant sur le premier car si l'on pouvait montrer qu'une taxe environnementale introduite en respectant la neutralité budgétaire est sans coût, il suffirait alors, pour justifier sa mise en place, de s'assurer que les bénéfices environnementaux en sont positifs.

De nombreuses études, théoriques ou empiriques, se sont attachées ces dernières années à mettre en évidence les conditions d'apparition de ce double dividende. Elles constituent une littérature abondante et extrêmement variée tant dans les méthodes employées que dans les résultats. Notre présentation de cette littérature suivra le plan suivant : dans la section 2, nous nous attacherons à définir précisément le concept de double dividende en replaçant les unes par rapport aux autres les 
différentes notions utilisées ; dans la section 3, nous présenterons le modèle canonique d'analyse qui a fondé la remise en question théorique de la possibilité d'obtention d'un dividende nonenvironnemental ; enfin la section 4 exposera des modèles plus complets et plus réalistes qui plaident en faveur de l'existence du double dividende.

\section{De l'importance de la définition du double dividende}

Depuis que la notion de double dividende a été invoquée pour rendre acceptables les écotaxes, elle l'a été sous différentes formes, parfois non cohérentes entre elles. Il est important de clarifier ce concept.

D'une façon générale on parlera de double dividende dans le cas où l'instauration (ou l'augmentation) d'une taxe environnementale, à recettes budgétaires inchangées pour l'État (neutralité budgétaire) fait apparaître non seulement un bénéfice environnemental mais aussi un deuxième bénéfice, de nature économique. Comme le note Ekins [1997], un tel bénéfice peut prendre plusieurs formes, compatibles ou non, qui correspondent aux différentes définitions utilisées :

- un dividende emploi lorsque le recyclage du rendement de la taxe permet la réduction du chômage ; c'est la notion à laquelle se réfèrent quasi-exclusivement les évaluations réalisées à l'aide de modèles macroéconométriques.

- un dividende d'efficacité lorsque la réforme fiscale accroît l'efficacité du système fiscal, en en réduisant les distorsions ( $c f$ encadré) ; c’est le concept utilisé par les études théoriques, effectuées le plus souvent dans un cadre d'équilibre général ; c'est aussi la définition défendue par Goulder [1994].

- un dividende redistributif (dit aussi parfois dividende social) lorsque le processus de redistribution choisi permet d'améliorer l'équité. En ce qui concerne ce troisième type de second dividende, Pearson et Smith [1991], Smith [1992] ainsi que Barker et Johnstone [1993] ont montré que les taxes sur le carbone pèsent relativement plus sur les ménages à faibles revenus et qu'en l'absence d'une redistribution appropriée, elles devraient conduire au contraire à des coûts supplémentaires en termes d'équité. 
La définition du concept de distorsion fiscale repose sur la comparaison entre le rendement de la taxe et la perte d'utilité provoquée par cette taxe pour le consommateuri. La perte de bien-être peut être mesurée soit par la variation équivalente, la variation de revenu qui serait nécessaire pour que l'individu atteigne le même niveau de bienêtre final, aux prix antérieurs à l'instauration de la taxe, soit par la variation compensatoire, la variation de revenu nécessaire pour que l'individu conserve son niveau de bien-être initial, aux prix atteints après l'instauration de la taxe.

Dans le cas d'un impôt forfaitaire, variation compensatoire et variation équivalente sont les mêmes : la charge en termes de bien-être est exactement égale au montant d'impôt prélevé. L'impôt forfaitaire n'entraîne qu'un effet de revenu et non un effet de substitution : c'est en ce sens qu'il est dit ne provoquer aucune distorsion. Dans le cas d'une accise prélevée sur l'un des biens consommés, le rendement de l'impôt prélevé est en revanche inférieur à la charge totale de l'impôt pour le consommateur. La différence entre les deux est la charge excédentaire de l'impôt (dite aussi perte sèche, ou coût social de l'impôt) provoquée par l'effet de substitution dû à l'accise qui induit ainsi une distorsion fiscale.

La charge excédentaire d'un impôt quelconque peut ainsi être envisagée de façon analogue comme l'équivalent monétaire de la perte de bien-être pour les consommateurs, à recette identique pour l'Etat, qui résulte du choix de cet impôt au lieu de l'impôt forfaitaire, ou comme la perte de recettes pour l'Etat, à perte de bien-être identique pour les consommateurs.

Il est ainsi clair qu'il ne sera possible d'obtenir un double dividende que si l'économie n'est pas initialement dans une situation optimale ${ }^{\mathrm{ii}}$ (et équitable), ce qui est le cas des économies occidentales. Différentes estimations de la charge excédentaire marginale du système fiscal américain suggèrent qu'elle est très importante (de l'ordre de 40 à 50 cents par dollar de recettes fiscales pour Ballard et al. [1985] ou Nordhaus [1991]). En l'absence d'études comparables pour l'Europe ou la France, on peut remarquer avec Ekins [1997] que les distorsions fiscales y sont au moins aussi importantes qu'aux USA, dans la mesure où le poids des prélèvements obligatoires est dans la Communauté européenne supérieur de 50\% à celui des États-Unis ou du Japon, et qu'il n'y a aucune raison de penser que le système fiscal européen soit plus efficace que le système fiscal américain.

C'est à la notion de dividende d'efficacité que s'intéresse Goulder [1994] (en faisant totalement abstraction des considérations d'équité), cette notion constituant désormais la référence commune à tous les travaux théoriques sur le sujet. Il considère en effet que la seule mesure rigoureuse du second dividende est l'évaluation en termes d'équivalents monétaires de pertes ou gains de bien-être social car c'est la seule mesure synthétique qui puisse être adoptée. Il conteste ainsi l'utilisation, dans de nombreux travaux empiriques, des variations du PIB, de la croissance du PIB ou de l'emploi comme mesure du second dividende (Carraro et alii [1996], Majocchi [1994], etc), 
expliquant que les résultats qu'ils en tirent sont souvent en contradiction apparente avec les résultats théoriques parce que "la question de savoir si une substitution fiscale, à dépenses publiques inchangées, entraîne des coûts positifs est une question différente de celle de savoir si elle entraîne une réduction du PIB ou de la croissance " (Goulder [1995a]).

Il est en outre désormais d'usage de distinguer, comme Goulder [1994], trois niveaux de l'hypothèse de double dividende :

$\diamond$ Version faible : Utiliser le rendement de la taxe environnementale pour financer des baisses de taux marginaux d'une taxe distordante existante permet d'en diminuer les coûts bruts par rapport au cas où le rendement de la taxe serait reversé aux contribuables sous forme forfaitaire (lump-sum). Cette version faible de l'hypothèse de double dividende est toujours vérifiée par définition même d'une taxe distordante : son existence implique une perte sociale en bien-être, charge excédentaire (excess burden), positive, alors qu'une taxe forfaitaire présente une charge excédentaire nulle et n'est donc pas distordante. Remplacer par une taxe environnementale la taxe distordante procure toujours un gain en bien-être par rapport à une redistribution forfaitaire du rendement ${ }^{\mathrm{iii}}$.

$\diamond$ Version intermédiaire : Il est possible de trouver une taxe distordante telle que la substitution de la taxe environnementale à cette taxe présente des coûts bruts nuls ou négatifs.

$\diamond$ Version forte : La substitution, sous la condition de neutralité budgétaire, d'une taxe environnementale à une taxe distordante typique ou représentative du système fiscal existant implique un coût brut (en termes de bien-être) nul ou négatif. Les formes fortes du double dividende sont ainsi vérifiées si la taxe environnementale est moins distordante que les taxes distordantes existantes, autrement dit si la taxe environnementale induit un coût en bien-être plus faible qu'une taxe distordante de même rendement. Dans la pratique, on parle de version forte du double dividende en regroupant versions intermédiaire et forte.

L'évaluation de ce second dividende est délicate dans la mesure où les différents impôts et taxes, distordants, interagissent : les coûts bruts (hors bénéfices environnementaux) de nouvelles taxes environnementales dépendent des taxes distordantes préexistantes (sur le même produit ou sur les autres marchés). Par ailleurs, lorsque la mise en place de la taxe environnementale est envisagée sous l'hypothèse de neutralité budgétaire (les mêmes dépenses publiques pouvant ainsi être financées), par substitution à d'autres taxes existantes, les coûts bruts de la taxe ne dépendent plus seulement de ses propres coûts directs mais aussi des bénéfices en termes d'efficacité procurés par la réduction 
d'autres taxes distordantes. Parry [1995] mettait ainsi en évidence le fait que l'apparition d'un second dividende dépend de l'amplitude respective de deux effets : l'effet de recyclage du revenu, qui permet de réduire la charge excédentaire des impôts préexistants, et l'effet d'interaction des taxes qui peut augmenter les coûts bruts en bien-être de la taxe environnementale.

\section{Les premiers modèles théoriques : une réfutation de l'hypothèse de double dividende}

Les premiers travaux analytiques dans un cadre d'équilibre général réfutent la version forte du double dividende : c'est le cas de Bovenberg et de Mooij [1994a], [1994b] et [1997b], Bovenberg et van der Ploeg [1994a], [1994b] et [1996], de Parry [1995] ou encore de Goulder [1995] et de Bovenberg et Goulder [1996]. Selon ces travaux, même si la taxe environnementale contribue à résoudre un problème environnemental, elle aggrave vraisemblablement les distorsions fiscales. Ces différentes contributions s'opposent ainsi aux opinions émises au début des années quatre-vingt-dix (par Oates [1991] ou Pearce [1991] par exemple) puisqu'elles semblent montrer que les distorsions existantes du système fiscal ne constituent pas un bon argument pour la mise en place d'une taxe environnementale. Au contraire, plus les coûts d'efficience sont élevés en raison des distorsions existantes, plus il faudrait que les bénéfices environnementaux potentiels soient élevés pour justifier les coûts supplémentaires dus à la taxe environnementale.

La source de ce courant d'analyse est constituée par les articles de Bovenberg et de Mooij [1994a] et [1994b] que nous allons utiliser comme trame ${ }^{\mathrm{iv}}$ pour l'exposition des résultats obtenus par des modèles d'équilibre général ainsi que pour la mise en évidence des éléments favorables, même dans ce cadre d'analyse, à l'obtention d'un double dividende.

Le modèle développé dans cet article est un modèle statique d'équilibre général d'une petite économie ouverte. Il fait apparaître deux distorsions : la distorsion environnementale associée aux effets externes de la pollution et une distorsion sur le marché du travail en raison de l'existence d'une taxe sur le travail. Les dépenses publiques sont supposées exogènes (au contraire de Bovenberg et van der Ploeg [1994b] qui supposent que les impôts et les dépenses publiques sont déterminés de façon optimale). Tous les biens, sauf le travail, sont supposés parfaitement mobiles entre pays ; par conséquent, dans le cadre choisi de petite économie ouverte, tous les prix sont exogènes (fixés par l'étranger) à l'exception du taux de salaire déterminé par l'équilibre sur le marché du travail.

Les émissions polluantes sont provoquées par la consommation d'un bien final polluant et par l'utilisation par les entreprises d'un input polluant. La technologie de production est supposée 
identique pour les deux types de biens de consommation : le bien propre et le bien polluant ; la fonction de production est à trois facteurs (travail, capital et input polluant tel que l'énergie), elle présente des rendements constants.

La fonction d'utilité du ménage représentatif dépend positivement de la consommation des deux biens de consommation, du loisir et de la qualité de l'environnement. On suppose le loisir faiblement séparable des deux biens finaux ; la qualité de l'environnement est un bien collectif, faiblement séparable des biens privés, ce qui implique que les modifications de qualité de l'environnement n'ont aucune influence ni sur l'arbitrage consommation-loisir ni sur la répartition de la consommation entre bien propre et bien polluant. Ceci permet une analyse séparée des différentes composantes du bien-être social : bien-être environnemental et non-environnemental.

Les taxes environnementales envisagées portent soit sur le bien de consommation polluant soit sur l'input polluant. Les effets en termes de bien-être de petites variations de la fiscalité sont mesurés par la charge excédentaire occasionnée par des augmentations des taxes environnementales spécifiques compensées par une réduction de la taxe ad valorem sur le travail. Cette charge excédentaire correspond à l'excès de perte de bien-être des consommateurs par rapport aux revenus collectés par l'état : elle peut être considérée comme la mesure des coûts cachés du financement des dépenses publiques. Le raisonnement est mené en variations relatives par rapport à l'équilibre, Bovenberg et de Mooij évaluent ainsi la charge excédentaire marginale par la variation compensatoire, c'est-à-dire par la variation de revenu qu'il faudrait procurer au ménage représentatif pour qu'il conserve son niveau initial d'utilité alors que l'on procède à une petite variation des taux de l'impôt $\mathrm{t}^{\mathrm{v}}$ : une valeur positive de cette charge excédentaire signifie une perte de bien-être à la suite de la réforme fiscale.

Bovenberg et de Mooij [1994b] font apparaître la charge excédentaire marginale comme la somme d'un effet de modification de la qualité de l'environnement (premier dividende) et d'un effet de modification de la base fiscale (second dividende éventuel). Intuitivement, l'effet de base fiscale représente les conséquences en termes de bien-être d'une combinaison différente des taux d'imposition sur l'efficacité du système fiscal en tant qu'instrument de prélèvement de revenus pour l'État : une érosion de la base fiscale indique que le système devient moins efficace puisqu'il faut appliquer des taux de taxes plus élevés pour collecter le même niveau de recettes.

Premier résultat : la création ex nihilo de taxes environnementales est bénéfique pour l'environnement et n'affecte nullement la capacité du système fiscal à prélever des recettes. Il n'y a par conséquent pas d'opportunité de double dividende. En effet, quelle que soit la taxe appliquée, la variation d'emploi est nulle, même si les mécanismes expliquant cette rigidité de l'emploi diffèrent 
selon la taxe choisie. Dans tous les cas, la variation de l'offre de travail résulte de celle du pouvoir d'achat du salaire net d'impôt. La taxe sur le bien de consommation polluant introduit un coin fiscal défini comme l'écart entre le salaire brut (versé par les entreprises) et le pouvoir d'achat du salaire net (reçu par les ménages) : dans ce cas, remplacer en partie la taxe distordante sur le salaire par une taxe environnementale (à dépenses publiques constantes) affecte seulement la composition du coin fiscal sans modifier l'ampleur globale de cette distorsion fiscale. En revanche la taxe sur l'input polluant diminue le taux de salaire brut mais cet effet est compensé sur le taux de salaire net par la baisse de l'impôt sur les salaires rendue possible à dépenses inchangées. Dans une petite économie ouverte, la substitution d'une taxe sur la pollution à une taxe sur le travail est équivalente à la substitution d'une taxe implicite à une taxe explicite sur le travail.

Deuxième résultat: en présence de taxes environnementales initiales, leur augmentation réduit à la fois l'emploi et la production si l'élasticité-salaire non compensée de l'offre de travail est positive. Cet effet est dû à la réduction du pouvoir d'achat du salaire net et donc à l'affaiblissement de l'incitation à offrir du travail : en effet, la diminution du taux de taxe sur le travail ne compense pas entièrement pour les travailleurs l'effet néfaste de la taxe environnementale sur leur pouvoir d'achat, en raison de l'érosion de la base fiscale résultant de la substitution, de bien propre au bien polluant, opérée par les consommateurs. Le remplacement de la taxe sur le travail par une taxe environnementale sur la consommation élargit le coin fiscal entre salaire brut et pouvoir d'achat du salaire net. Dans le cas d'une taxe sur l'input polluant, la diminution du pouvoir d'achat du salaire net est initiée en revanche par une baisse des salaires bruts accompagnée de l'érosion de la base fiscale de la taxe sur l'énergie car la demande en est diminuée.

L'ampleur de l'effet négatif sur l'emploi, et donc sur le bien-être non environnemental, dépend de l'élasticité de l'offre de travail mais aussi d'autres facteurs importants. De fortes élasticités de substitution entre bien (ou input) propre et polluant augmentent à la fois les effets positifs sur la qualité de l'environnement et les effets négatifs sur les incitations au travail, en renforçant l'érosion de la base de la taxe qui limite la réduction possible de la taxe sur les salaires. Un taux initialement élevé de taxe environnementale renforce encore cet effet-revenu négatif de l'érosion de la base fiscale. Enfin, plus la taille du secteur public est importante (soit le montant de dépenses publiques à financer) et plus les taux de taxe initiaux doivent être élevés, ce qui limite l'efficacité de la taxe environnementale à prélever des recettes fiscales supplémentaires (cette efficacité dépendant de l'ampleur des pertes de revenus provoquées par des modifications de comportement des consommateurs - substitutions bien propre / bien polluant et loisir / consommation - qui érodent la base des impôts existants). 
Ce modèle aboutit par conséquent à la conclusion marquante qu'il n'y a pas de présomption d'existence de double dividende, au sens fort de Goulder [1994]. Ce résultat tient à la nature du problème : la qualité de l'environnement est un bien public dont chaque résident bénéficie quelle que soit la quantité de travail qu'il offre, tandis que l'amélioration de cette qualité est financée par le seul facteur de production immobile, soit le travail, qui supporte ex post toutes les taxes nécessaires au financement des dépenses publiques. En outre, la taxe environnementale ne taxe pas le travail efficacement puisqu'en plus de la réduction du pouvoir d'achat du revenu salarial qu'elle provoque, elle distord aussi la composition du panier de consommation ou le processus de production ; la taxe réduit la pollution en incitant les contribuables à éviter l'impôt, ce qui contraint l'État à choisir des taux de taxe plus élevés pour pouvoir financer ses dépenses. Il y a dans cette situation une incompatibilité entre dividende environnemental et second dividende.

Ces résultats sont généralisés par Bovenberg et van der Ploeg [1994a] au cas où les dépenses publiques sont endogènes. Ils décomposent alors le bien-être social en trois composantes : bien-être vert (environnemental), bien-être public ou rouge (fonction de la consommation publique) et bien-être bleu (privé, croissant avec l'emploi dans ce modèle). Le second dividende au sens de Goulder est donc scindé en dividende public (qui était nul dans le cas d'exogénéité des dépenses publiques) et dividende emploi (bleu). Le système fiscal initial est supposé optimal, la pollution provient uniquement de la consommation et l'augmentation de la taxe environnementale est envisagée en réponse à une plus forte sensibilisation des agents à la qualité de l'environnement. Le résultat essentiel de cet article est que l'augmentation de la taxe sur la consommation de bien polluant conduit à une diminution du niveau d'emploi (il n'y a donc pas de dividende emploi) même si elle permet une réduction de la taxe sur le travail et ceci quel que soit le signe de l'élasticité non compensée de l'offre de travail au salaire net. Les mécanismes sont les mêmes que précédemment : si la substitution est aisée entre consommations de bien polluant et de bien propre, la taxe induit une déformation de la structure de la consommation privée favorable à la qualité de l'environnement. Ceci érode la base fiscale, le système d'imposition devient moins efficace pour obtenir le même montant de recettes fiscales et la baisse de la taxe sur le salaire est alors insuffisante pour compenser la détérioration du pouvoir d'achat du salaire par la taxe environnementale. Les ménages se tournent alors vers le loisir pour échapper à l'impôt ce qui aggrave l'effet d'érosion de la base fiscale et les distorsions préexistantes sur le marché du travail. Il est alors optimal pour l'État de diminuer ses dépenses : le bien-être public est évincé par l'augmentation de la sensibilité environnementale. 
Les dividendes vert et public peuvent cependant être compatibles si l'élasticité de substitution entre consommation et loisir est suffisamment grande et si, en outre, les biens polluant et propre sont faiblement substituables et enfin si la productivité de l'activité publique de dépollution décroît avec le niveau de l'abattement de pollution réalisé ; en effet, dans ce cas l'amélioration de la qualité de l'environnement est obtenue davantage par la diminution de l'emploi et de la production que par une composition moins polluante de la production totale. Mais alors, c'est le dividende emploi qui ne peut être obtenu.

\section{Les analyses plus récentes : la mise en évidence de conditions plausibles d'occurrence du double dividende}

Goulder [1994] analyse les raisons de l'inexistence du double dividende dans les modèles à la Bovenberg et de Mooij et précise un certain nombre de conditions qui permettent d'augmenter la probabilité d'obtention d'un double dividende. Tout d'abord le système fiscal initial doit être fortement sous-optimal, ce qui se traduit par de grandes différences entre les charges excédentaires marginales des différents impôts et taxes. De plus, le poids de la taxe environnementale doit porter $e x$ post sur un bien ou un facteur sur lequel pesait une charge excédentaire marginale relativement faible : ainsi la distorsion supplémentaire créée par la taxe environnementale est la moins forte possible. Par exemple, aux États-Unis où la taxe sur le travail est moins distordante que celle sur le capital, c'est une substitution de la taxe environnementale à la taxe sur le capital qui a le plus de chances de conduire à un double dividende tandis qu'en Europe, il semble que ce soit au contraire une réduction des prélèvements portant sur le travail qui soit le plus efficace. Enfin la taxe environnementale doit avoir l'assiette la plus large possible afin de ne pas induire des distorsions sur d'autres marchés ce qui réduirait le bien-être.

Finalement, comme le résume Ligthart [1998], la condition essentielle pour qu'un double dividende au sens fort puisse être obtenu est que la charge de la taxe puisse être transférée sur d'autres facteurs de production ou d'autres catégories de ménages. C'est ce que montrent un certain nombre d'études postérieures à celle de Bovenberg et de Mooij [1994] (dans lequel le revenu salarial des ménages supporte l'intégralité de la charge fiscale). Le déplacement de la charge fiscale peut être dirigé vers trois types de supports : les facteurs fixes de production, les ménages qui consomment grâce à des revenus de transfert (comme les retraités ou les chômeurs), les pays étrangers qui ne pourraient pas contrôler leurs termes de l'échange. 
Par ailleurs, le rôle essentiel joué dans les résultats obtenus par l'élasticité non compensée de l'offre de travail au pouvoir d'achat du salaire met l'accent sur des hypothèses essentielles de l'analyse en termes d'équilibre général menée par Bovenberg et de Mooij, à savoir la concurrence pure et parfaite et l'équilibre sur le marché du travail. Lever ces hypothèses permet de montrer la possibilité d'obtenir un double dividende, comme en témoignent un certain nombre d'études que nous allons maintenant présenter.

\section{Le rôle des facteurs fixes de production}

Bovenberg et van der Ploeg [1996] ainsi que Ligthart et van der Ploeg [1996] développent des modèles dans lesquels le processus productif repose sur trois facteurs substituables (le travail, le capital qui est le seul facteur fixe, et une ressource polluante comme l'énergie). Le salaire est supposé rigide et fixé à un niveau excessif pour équilibrer le marché du travail, faisant apparaître ainsi du chômage involontaire ${ }^{\mathrm{vi}}$. Dans ce cas, un double dividende au sens fort peut être obtenu lors de l'instauration d'une taxe environnementale si la part du facteur fixe est suffisamment importante dans la valeur ajoutée et si le travail est un meilleur substitut à l'énergie que ne l'est le capital. En effet, dans ce cas, l'amélioration de la qualité de l'environnement est obtenue par l'adoption d'une technique de production plus intensive en travail au lieu que ce soit, comme dans le modèle précédent, par une diminution de la production. Ces conditions assurent que la charge de la taxe sur la pollution est déplacée du travail vers les détenteurs du capital. Mais si le capital est mobile internationalement, il peut alors “échapper" à la taxation par la délocalisation et le second dividende ne peut être obtenu (Bovenberg et van der Ploeg [1994c]).

\section{Le rôle du déplacement de la charge fiscale sur d'autres catégories de ménages}

Le modèle de Bovenberg [1997] supposent les agents hétérogènes : les uns sont salariés, les autres reçoivent uniquement des transferts de l'État (agents inactifs : chômeurs ou retraités). Lorsque l'on instaure ou augmente la taxe sur les biens de consommation polluants, la charge fiscale repose initialement sur l'ensemble des consommateurs. L'effet premier est une réduction du pouvoir d'achat de tous les consommateurs qui conduit à une détérioration de l'emploi car les actifs substituent du loisir à la consommation polluante. Mais la neutralisation budgétaire de la taxe par une réduction des taxes portant sur le travail réduit les distorsions sur le marché du travail et est donc favorable à l'emploi sans bénéficier aux ménages inactifs dont le pouvoir d'achat du revenu diminue. La charge fiscale se déplace ainsi des actifs aux inactifs parce que l'assiette de la taxe environnementale est plus 
large que celle de la taxe sur le travail. Si cet effet de déplacement de la charge l'emporte sur le premier effet, un second dividende apparaît. Il faut noter qu'ici ce second dividende n'est que d'efficacité-emploi car la satisfaction des inactifs se détériore, il est donc incompatible avec la recherche de l'équité entre agents.

\section{Le rôle du déplacement de la charge fiscale sur l'extérieur}

Goulder [1994] remarque qu'en économie ouverte, un pays peut reporter une partie de la charge fiscale environnementale sur l'extérieur en utilisant la taxe sur la pollution comme un moyen d'améliorer ses termes de l'échange. En effet, si ce pays est importateur net du produit polluant auquel s'applique la taxe (l'énergie par exemple) et si son poids dans le marché mondial est suffisamment important pour qu'il puisse exercer un pouvoir de monopsone, même relatif, la taxation du produit polluant importé diminue sa demande nationale en bien polluant dont le prix mondial hors-taxe est alors réduit. Si le rendement de la taxe est affecté à la réduction de taxes distordantes pesant sur les agents nationaux, l'amélioration des termes de l'échange reporte sur l'étranger une partie de la charge de la taxe. Des gains de termes de l'échange suffisamment grands peuvent permettre de compenser les coûts bruts de la taxe et faire apparaître, au niveau national, un fort double dividende. Ainsi, les pays exportateurs de pétrole supporteraient-ils, via la détérioration de leurs termes de l'échange, la charge d'une taxe sur l'énergie ou sur le carbone à la place des consommateurs d'énergie des pays importateurs de pétrole (Ligthart [1998]). Une fois de plus, l'objectif d'efficacité s'avère incompatible avec celui d'équité, au niveau international cette fois.

\section{Le rôle du chômage involontaire}

Nous avons vu plus haut que dans le modèle de Bovenberg et van der Ploeg [1995], où le chômage involontaire est lié à l'existence d'un salaire rigide et trop élevé, supposé exogène, la possibilité d'apparition d'un double dividende tient à l'effet de déplacement de la charge fiscale due à la taxe environnementale sur le capital et non à l'existence de chômage involontaire.

D'autres articles comme celui de Schneider [1997] lient en revanche au chômage involontaire la possibilité d'obtention d'un double dividende, sous l'hypothèse d'endogénéité du salaire. Le modèle repose sur une analyse du marché du travail dans le cadre de la théorie du salaire d'efficience selon Solow. La productivité du travail dépend de l'effort fourni par les salariés, effort supposé croissant avec le salaire. Dans ce cas de figure, le chômage involontaire apparaît en raison de la fixation par les entreprises d'un taux de salaire incitatif supérieur à celui qui équilibrerait le marché du 
travail (la minimisation du coût du travail par l'entreprise conduit à un salaire optimal tel que l'élasticité de la fonction d'effort par rapport au salaire est unitaire). Dans un tel modèle, l'emploi et le salaire évoluent de pair car la réduction du chômage conduit à une diminution de l'effort fourni par les salariés, ce que compensent les entreprises par un salaire plus élevé. La pollution est supposée due à l'utilisation par le système productif d'un input polluant. L'instauration d'une taxe sur l'énergie a un effet négatif sur l'utilisation de tous les facteurs de production par les entreprises mais la réduction de la taxe de travail peut contrebalancer cet effet sur l'emploi. En effet, une plus faible pression fiscale sur le travail permet à l'entreprise d'offrir un salaire plus faible sans modifier le niveau d'effort des salariés et donc leur productivité : le taux de chômage diminue si le niveau d'effort, et donc le salaire, sont faiblement élastiques au taux de chômage (ce qui sera d'autant plus vraisemblable que le taux de chômage initial est fort) afin de ne pas annihiler la baisse du coût du travail. En outre, les gains en bien-être de la réforme fiscale seront d'autant plus élevés que les distorsions sont initialement fortes sur le marché du travail.

Les cas où les salaires sont fixés selon un processus de négociations salariales correspondent de nouveau à des situations où le double dividende apparaît en raison du report de la charge fiscale sur certains agents. Bovenberg et van der Ploeg [1994a] supposent que les salaires sont fixés par application d'un taux de mark-up sur la productivité du travail dans le cadre d'un modèle d'appariement (dans Brunello [1996], les salaires sont déterminés par négociations entre firmes et syndicats). Les indemnités chômage sont supposées être indexées sur le taux de salaire des employés. La hausse de la taxe environnementale détériore le pouvoir d'achat des salariés comme des chômeurs mais ces derniers ne bénéficiant pas de la compensation par l'abaissement de la taxe sur le travail, ils supportent l'essentiel de la charge fiscale. Le double dividende résulte ici encore d'une détérioration de l'équité.

\section{Le rôle de la concurrence imparfaite sur les marchés des biens}

L'influence de l'imperfection de la concurrence sur l'obtention d'un double dividende est mise en évidence par Marsiliani et Renström [1997] dans le cadre d'une économie fermée et par Holmlund et Kolm [1997] pour une économie ouverte. La pollution est supposée être provoquée par l'utilisation de l'énergie comme facteur de production et comme bien de consommation. Les firmes sont en situation de concurrence monopolistique et les salaires sont fixés par négociations entre syndicats et 
entreprises. Marsiliani et Renström montrent que l'hypothèse de double dividende est vérifiée quels que soient le pouvoir de marché des firmes et le pouvoir de négociation des syndicats mais que le second dividende, l'augmentation de l'emploi, est d'autant plus important que les marchés sont peu concurrentiels (l'érosion de la base fiscale est en effet plus faible qu'en concurrence parfaite car on montre que la part de la consommation totale d'énergie par rapport au revenu du travail augmente avec l'imperfection des marchés).

\section{L'importance des hypothèses de séparabilité dans le bien-être des ménages}

L'hypothèse des modèles de Bovenberg et de Mooij [1994a] et [1994b] selon laquelle la qualité de l'environnement est un bien faiblement séparable des biens privés, donc sans influence ni sur l'arbitrage consommation-loisir ni sur la répartition de la consommation entre bien propre et polluant, est une hypothèse assez restrictive. Comme le note de Mooij [1997], même si l'hypothèse de séparabilité ne peut être en général rejetée, il est des cas où la nature de l'externalité (les congestions par exemple) rend difficilement imaginable que les ménages ne réagissent pas dans leurs comportements à des modifications de la qualité de l'environnement. C'est la raison pour laquelle il s'attache à évaluer l'importance de cette hypothèse de séparabilité dans le diagnostic de double dividende.

L'auteur montre que si le loisir est un meilleur substitut que la consommation totale pour la qualité de l'environnement, les améliorations de celle-là stimulent les incitations au travail en évinçant davantage le loisir que la consommation. Or dans ce modèle, le bien-être varie avec l'emploi. Par conséquent, plus le loisir et la qualité de l'environnement sont de relatifs substituts et plus le bienêtre non-environnemental est amélioré (ce qui vérifie l'hypothèse de double dividende fort) tandis que si le loisir et la qualité de l'environnement sont de relatifs compléments, la taxe environnementale exacerbe les distorsions sur le marché de travail, niant alors la possibilité d'un double dividende au sens fort.

Enfin, dans le cas où la qualité de l'environnement affecte l'arbitrage entre consommation de bien propre et consommation de bien polluant (Beaumais et Schubert [1994]), le diagnostic est un peu plus délicat : c'est le bénéfice environnemental lui-même qui est à l'origine d'une partie du bénéfice non-environnemental éventuel, qui ne provient plus seulement de l'effet de recyclage du revenu.

\section{Le rôle de la qualité de l'environnement comme facteur de productivité}


Partant de la constatation que la pollution peut provoquer des pertes de productivité, et suivant ainsi une suggestion de Goulder [1994], Bovenberg et de Mooij [1997] introduisent dans un modèle de croissance la qualité de l'environnement comme input productif, agissant comme une externalité positive, dans un processus mettant en jeu le travail, le capital et un input polluant tel que l'énergie.

L'effet sur la croissance d'une augmentation de la taxe sur l'input polluant dépend de son impact sur le taux de rendement net après impôt qui conditionne les incitations à épargner. Or l'effet net sur la productivité marginale du capital résulte de deux effets contraires. D'un côté, un taux de taxe sur la pollution plus élevé diminue la productivité marginale du capital en réduisant la contribution de l'input polluant : les coûts de mise en œuvre de la taxe environnementale dépendent de l'élasticité de la production à la pollution. De l'autre côté, l'amélioration de la qualité de l'environnement augmente la productivité de tous les facteurs : ce bénéfice environnemental augmente avec l'importance de l'externalité dans la combinaison productive et avec la sensibilité de la qualité de l'environnement à une réduction de la pollution. Si le premier effet l'emporte sur le second, l'augmentation de la taxe environnementale réduit le taux de rendement net du capital et donc détériore la croissance (c'est le cas dans les modèles n'intégrant pas d'externalité positive de l'environnement sur la productivité, comme Bovenberg et de Mooij [1994a] et [1994b]). En revanche, si le second effet l'emporte sur le premier, c'est-à-dire si l'externalité de l'environnement est suffisamment forte, une taxe environnementale peut non seulement améliorer la qualité de l'environnement mais aussi élever le bien-être non-environnemental en stimulant la croissance. Du point de vue de Goulder cependant, ce résultat, à proprement parler, ne correspond pas à la notion de double dividende au sens fort puisqu'il tient compte d'effets du côté des bénéfices environnementaux qu'il est difficile ici de séparer des coûts bruts économiques de la taxe.

Enfin, de Mooij [1997] considère le cas où la qualité de l'environnement intervient directement de façon non-séparable dans la technologie de production en élevant la productivité du travail. Il montre alors que les variations du bien-être dépendent des valeurs relatives des élasticités de substitution de la fonction de production. Notamment, si l'élasticité de substitution d'Allen entre l'input polluant et le travail efficace (agrégat travail-environnement) l'emporte sur l'élasticité de substitution entre le travail et la qualité de l'environnement (et tout particulièrement si ces derniers sont complémentaires ou quasiment), un environnement plus propre incite à adopter des techniques intensives en travail : la taxe environnementale peut alors, en augmentant l'emploi, conduire à un double dividende au sens fort. 


\section{Conclusion}

Les premiers travaux théoriques menés sur le thème du double dividende étaient en contradiction avec les premières évaluations empiriques : tandis que celles-là mettaient en évidence la possibilité d'obtenir un double dividende, sous la forme d'une réduction du chômage, ceux-ci aboutissaient à la négation de cette possibilité de double dividende. Les différentes définitions utilisées pour ce concept ne sont pas vraiment responsables de cette contradiction : en effet, le second dividende, défini comme la variation de bien-être non-environnemental, varie avec le niveau d'emploi et ainsi le second dividende est le plus souvent un dividende emploi. La raison principalement invoquée pour expliquer cette divergence est plutôt la différence d'hypothèses théoriques et l'opposition entre modèles macroéconométriques et modèles d'équilibre général. Nous avons vu que même dans ce cadre, la possibilité de déplacer la charge fiscale sur le capital ou d'autres agents que les salariés, ou la prise en compte du rôle de la qualité de l'environnement dans les comportements des ménages ou dans la structure productive permettent de faire apparaître un double dividende. Enfin, le relâchement de l'hypothèse de concurrence pure et parfaite sur les marchés ainsi que la considération de l'existence de chômage involontaire rendent également plus probable l'occurrence d'un double dividende au sens fort.

\section{Bibliographie}

Assouline M., Lemiale L. [1998], “Théorie des externalités : les instruments des politiques de l'environnement" in Schubert K. et Zagamé P., L'environnement - Une nouvelle dimension de l'analyse économique, Vuibert, Paris, 1998.

Ballard C.I., Shoven J.B., Whalley J. [1985], “"General Equilibrium Computations of the Marginal Welfare Costs of Taxes in the United States ", American Economic Review, 75, n 1, p. 128-138.

Barker T., Johnstone N. [1993], "Equity and Efficiency in Policies to Reduce Carbon Emissions in the Domestic Sector ”, Energy and Environment, 4, n 4, p. 335-361.

Baumol W.J., Oates W.E. [1988], The Theory of Environmental Policy, Cambridge University Press, 2nd edition.

Beaumais O, Godard O. [1994], “Économie, croissance et environnement. De nouvelles stratégies pour de nouvelles relations", Revue Économique, 44, Hors série "Prospectives et réflexions stratégiques à moyen terme, p. 143-176.

Beaumais O, Schubert K. [1994], “Équilibre général et environnement : de nouveaux comportements pour le consommateur et le producteur", Revue Économique, 44, Hors série "Prospectives et réflexions stratégiques à moyen terme, p. 905-916.

Beaumais O., Schubert K., Zagamé P. [1998], “Les outils de l'évaluation ” in Schubert K. et Zagamé P., L'environnement - Une nouvelle dimension de l'analyse économique, Vuibert, Paris, 1998. 
Bovenberg A.L. [1997], “Environmental Policy, Distortionary Labour Taxation and Employment : Pollution Taxes and the Double Dividend" in Carraro C., Siniscalco D.., New Directions in the Economic Theory of the Environment, Cambridge University Press, 1997.

Bovenberg A.L., de Mooij R.A. [1994a], “"Environmental Levies and Distortionary Taxation ”, American Economic Review, 84, n 4, p. 1085-1089.

Bovenberg A.L., de Mooij R.A. [1994b], “Environmental Taxes and Labor-Market Distortions”, European Journal of Political Economy, 10, p. 655-683.

Bovenberg A.L., de Mooij R.A. [1996], "Environmental Taxation and the Double-Dividend : The Role of Factor Substitution and Capital Mobility" in Carraro C., Siniscalco D.., Environmental Fiscal Reform and Unemployment, Kluwer Academic Publishers, 1996.

Bovenberg A.L., de Mooij R.A. [1997a], “Environmental Tax Reform and Endogenous Growth”, Journal of Public Economics, 63, p. 207-237.

Bovenberg A.L., de Mooij R.A. [1997b], "Environmental Levies and Distortionary Taxation : Reply", American Economic Review, 87, $\mathrm{n}^{\circ}$ 1, p. 252-253.

Bovenberg A.L., Goulder L.H. [1996], “ Optimal Environmental Taxation in the Presence of Other Taxes : General Equilibrium Analysis", American Economic Review, 86, n 4, p. 985-1000.

Bovenberg A.L., Goulder L.H. [1997], " Costs of Environmentally Motivated Taxes in the Presence of Other Taxes : General Equilibrium Analyses”, National Tax Journal, L, n 1, p. 59-87.

Bovenberg A.L., van der Ploeg F. [1994a], “Environmental Policy, Public Finance and the Labour Market in a Second-Best World”, Journal of Public Economics, 55, n 3, p. 349-390.

Bovenberg A.L., van der Ploeg F. [1994b], " Environmental Policy, Public Goods and the Marginal Cost of Public Funds", The Economic Journal, 104, March, p. 444-454.

Bovenberg A.L., van der Ploeg F. [1994c], " Green Policies and Public Finance in an Open Economy", Scandinavian Journal of Economics, 96, p. 343-363.

Bovenberg A.L., van der Ploeg F. [1996], “ Optimal Taxation, Public Goods and Environmental Policy with Unvoluntary Unemployment”, Journal of Public Economics, 62, p. 52-83.

Brunello. [1996], "Labor Market Institutions and the Double Dividend Hypothesis : An Application of the WARM Model" in Carraro C., Siniscalco D.., Environmental Fiscal Reform and Unemployment, Kluwer Academic Publishers, 1996.

Carraro C., Galeotti M., Gallo M. [1996], “Environmental Taxation and Unemployment : Some Evidence on the "Double Dividend Hypothesis" in Europe ", Journal of Public Economics, 62, p. 141-181.

CGP [1993], L'économie face à l'écologie, Rapport de l'Atelier I “Environnement, économie, croissance ” de la Commission "Environnement, croissance, qualité de vie" du Xième Plan, Éditions La Découverte / La Documentation française.

EC [1992], “The Climate Change - Economic Aspects of Limiting $\mathrm{CO}_{2}$ Emissions ”, European Economy, 52.

EC [1994], “Taxation, Employment and Environment : A Fiscal Reform for Reducing Unemployment”, study n³ in "Annual Economic Report for 1994 ”, European Economy, 56.

Ekins P. [1997], “ On The Dividends from Environmental Taxation" in O'Riordan T., Ecotaxation, Earthscan Publications, 1997.

GIEC (Groupe d'Experts Intergouvernemental sur l'Évolution du Climat) [1996], Le changement climatique, Dimensions économiques et sociales, 4D, Paris.

Goulder L.H. [1994], "Environmental Taxation and the "Double Dividend" : A Reader's Guide", NBER Working Paper, $\mathrm{n}^{\circ} 4896$.

Goulder L.H. [1995], “Effects of Carbon Taxes in an Economy with Prior Tax Distortions : An Intertemporal General Equilibrium Analysis”, Journal of Environmental Economics and Management, 29, p. 271-297. 
Holmlund B, Kolm A.S. [1997], “Environmental Tax Reform in a Small Open Economy With Structural Unemployment”, Working Paper, Department of Economics, Uppsala University.

Lee D.R., Misiolek W.S. [1986], “ Substituting Pollution Taxation for General Taxation : Some Implications for Efficiency in Pollution Taxation”, Journal of Environmental Economics and Management, 13, p. 338-347.

Lemiale L., Zagamé P [1998], “Taxation de l'énergie, efficience énergétique et nouvelles technologies : les effets macroéconomiques pour six pays de l'Union européenne" in Schubert K. et Zagamé P., L'environnement - Une nouvelle dimension de l'analyse économique, Vuibert, Paris, 1998.

Ligthart J.E. [1998], “ The Macroeconomic Effects of Environmental Taxes : A Closer Look at the Feasibility of "Win-Win " Outcomes", Working Paper of the International Monetay Fund, Washington, May 1998.

Ligthart J.E., van der Ploeg F. [1996], “ Optimal Government Policy, the Environment, Employment and Tax Shifting" in Carraro C., Siniscalco D.., Environmental Fiscal eform and Unemployment, Kluwer Academic Publishers, 1996.

Majocchi A. [1994], “The Employment Effects of Eco-Taxes: a Review of Empirical Models and Results " , OECD Workshop on Implementation of Environmental Taxes, Paris, 14--15 février.

Marsiliani L., Renström T. [1997], “ Imperfect Competition, Labour Market Distortions and the Double Dividend Hypothesis”, Discussion Paper, University of Birmingham, dec 1997.

de Mooij R.A. [1997], “The Double Dividend in Case of Environment-Economy Interactions” , CPB Discussion Paper (draft version), Workshop on Modelling Feedback of Externalities in General Equilibrium Models, Katholic University of Leuven, 20 juin.

Nordhaus W. [1991], “ To Slow or Not to Slow : the Economics of the Greenhouse Effect”, Economic Journal, 101, July, p. 920-937.

Oates W.E. [1991], “ Pollution Charges As A Source of Public Revenues”, Resources of the Future Discussion Paper, QE92-05.

Parry I.W.H. [1995], “ Pollution Taxes and Revenue Recycling”, Journal of Environmental Economics and Management, 29, $\mathrm{n}^{\circ}$ 3, p. S64-S77.

Parry I.W.H., Williams R.C.III, Goulder L.H. [1996], “When Can Carbon Abatement Policies Increase Welfare ? The Fundamental Role of Distorted Factor Markets", Resources for the Future Working Paper, Washington.

Pearce D.W. [1991], “ The Role of Carbon Taxes in Adjusting To Global Warming”, The Economic Journal , 101, p. 938-948.

Pearson M., Smith S. [1991], “ The European Carbon Tax : An Assessment of the EC's proposals”, Institute of Fiscal Studies, London.

Poterba J.M. [1993], “ Global Warming Policy : A Public Finance Perspective”, Journal of Economic Perspectives, 7, $\mathrm{n}^{\circ}$ 4, p. 47-63.

Proost S., Van Regemorter. [1995], “The Double-Dividend and the Role of Inequality Aversion and Macroeconomic Regimes ”, International Tax and Public Finance, 2, n 2, p. 207-219.

Schneider K. [1997], “ Involontary Unemployment and Environmental Policy : The Double Dividend Hypothesis", Scandinavian Journal of Economics, 99, n 1, p. 45-59.

Smith S. [1992], “ Taxation and the Environment : A Survey”, Fiscal Studies, 13, p. 21-57.

Terkla D. [1984], “ The Efficiency Value of Effluent Tax Revenues”, Journal of Environmental Economics and Management, 11, p. 107-123.

\footnotetext{
${ }^{\mathrm{i}}$ Le lecteur pourra se reporter, pour plus de précisions, à Wolfelsperger [1995].
} 
ii Certains auteurs supposent optimal le système fiscal initial et parlent alors de double dividende social si la taxe environnementale permet simultanément une amélioration de la qualité de l'environnement et une augmentation des dépenses publiques (Ligthart et van der Ploeg [1996]).

iii C'est du moins vrai dans le cas d'agents homogènes. En revanche, Proost et van Regemorter [1995] montrent que même la version faible du double dividende n'est pas vérifiée dans le cas d'agents hétérogènes, qui diffèrent par leur productivité du travail, leur probabilité d'être employés et leur patrimoine : un recyclage du rendement de la taxe par des transferts forfaitaire peut être plus efficace en termes de bien-être social (et plus équitable) qu'un recyclage par la diminution des cotisations sociales employeurs car dans le premier cas, le bien-être de tous les agents est amélioré tandis que dans le second, seul celui des salariés l'est.

${ }^{\text {iv }}$ Une présentation plus détaillée de ce modèle est donnée dans Assouline et Lemiale [1998].

${ }^{v}$ Pour des variations infinitésimales des taux d'imposition, cette variation compensatoire est égale à la variation équivalente, soit la variation de revenu que devrait subir le consommateur pour atteindre, aux prix de l'équilibre initial, le niveau d'utilité de l'équilibre final.

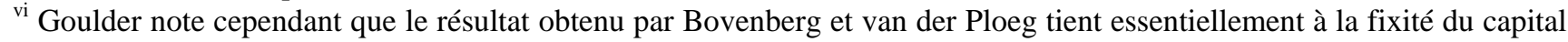
et qu'il serait le même en l'absence de chômage involontaire. 
This document was created with Win2PDF available at http://www.daneprairie.com. The unregistered version of Win2PDF is for evaluation or non-commercial use only. 